\title{
Terbinafine inhibits KSR1 and suppresses Raf-MEK-ERK signaling in oral squamous cell carcinoma cells
}

\author{
B. $\mathrm{LI}^{1}$, L. LU' ${ }^{2, *}, \mathrm{M} . \mathrm{ZHONG}{ }^{3}$, X. X. TAN ${ }^{1}$, C. Y. LIU', Y. GUO ${ }^{3}, \mathrm{X} . \mathrm{YI}^{1}$ \\ ${ }^{1}$ Department of oral anatomy and physiology, School of Stomatology, China Medical University, Shenyang 110002, Liaoning Province, P. R. China; \\ ${ }^{2}$ Department of oral and maxillofacial surgery, School of Stomatology, China Medical University, Shenyang 110002, Liaoning Province, P. R. \\ China; ${ }^{3}$ Central laboratory, School of Stomatology, China Medical University, Shenyang 110002, Liaoning Province, P. R. China
}

*Correspondence: libo919_oral@163.com

Received September 12, 2012 / Accepted January 30, 2013

\begin{abstract}
Terbinafine inhibits the proliferation of many types of cancer cells, but the underlying mechanism remains to be determined. By computer simulation, we found that kinase suppressor of Ras 1 (KSR1) is a possible target of terbinafine. Treatment of human oral squamous cell carcinoma (OSCC) KB cells with either terbinafine or siRNA to knockdown KSR1 reduced proliferation and induced apoptosis, which was accompanied by suppression of the Raf-MEK-ERK pathway. In vivo, KSR1 expression was significantly associated with the clinical staging of OSCC and the smoking habit of patients. Kaplan Meyer survival analysis demonstrated that the cumulative survival time of patients without KSR1 expression was significantly longer than those with KSR1 overexpression. Our data provide the basis for developing terbinafine to treat OSCC.
\end{abstract}

Key words: terbinafine, OSCC, apoptosis, KSR1, RAF-MEK-ERK signaling

Oral squamous cell carcinoma (OSCC) is the sixth most common human malignancy worldwide, accounting for 263,900 new cases and over 128,000 deaths in 2008 [1]. Thus, it is urgent to develop efficient treatments for this disease. Smoking, alcohol use, smokeless tobacco products, and HPV infections are the main risk factors for OSCC [2-5]. Most importantly, alterations in oncogenes and suppressor genes play an important role in OSCC [6]. The ras gene is one of the most frequently mutated oncogenes in oral cancer [7]; however, the function of kinase suppressor of Ras 1 (KSR1) in OSCC is largely unknown. KSR1, a 97-kDa protein, was originally identified in D. melanogaster and C. elegans as a positive modulator of the Ras/MAPK signaling pathway [8]. The Raf/MEK/ERK pathway plays a key role in the development and progression of cancer by regulating proliferation, transformation, differentiation, and apoptosis [9]. Overexpression of KSR1 in mouse

Abbreviations: TB, terbinafine; OSCC, Oral squamous cell carcinoma; KSR1, Kinase Suppressor of Ras1; DMSO, dimethyl sulfoxide; TBST, Tris Buffered Saline with Tween; MTT, 3-(4,5-dimethylthiazolyl)-2,5-diphenyltetrazoliumbromide; MEK, mitogen-activated protein/ extracellular signal-regulated kinase kinases; ERK, extracellular-signal-regulated kinase; HPV, human papillomavirus; C-TAK1, Cdc25 C-associated kinase 1; PDB, Protein Data Bank; siRNA, small interfering RNA. embryonic fibroblasts results in maximal assembly of the Raf-1/MEK/ERK/MAPK complex, and hence enhanced ERK/ MAPK signaling [10]. In addition, a recent study demonstrated that KSR1 expression is elevated in endometrial carcinoma [11]. These facts suggest that KSR1 may be a potential target for the development of cancer therapeutics.

Terbinafine (TB) is a generic antifungal agent used to treat superficial mycoses [12-13]. Recent studies have demonstrated its antitumor effects in many cancer cells, such as human colorectal (HT29 and COLO205) cells [16], human promyelocytic leukemia (HL60) cells [17], and human OSCC KB cells [18]. In the OSCC cell lines, SAS and SCC15, terbinafine inhibited the cell growth and proliferation accompanied with cell-cycle arrest, but the underlying molecular mechanism is unknown [18]. In this study, we found that terbinafine suppressed Raf-MEK-ERK signaling by targeting KSR1, which is overexpressed in OSCC. Our data suggest that targeting KSR1 is a promising therapeutic strategy for OSCC.

\section{Patients and methods}

Cells. The human OSCC cell line KB was obtained from the American Type Culture Collection (Rockville, MD, USA), and 
was grown in Dulbecco's Modified Eagle's Medium (DMEM, Hyclone, Logan, UT, USA) supplemented with $10 \%$ fetal bovine serum and antibiotics $(100 \mu \mathrm{M}$ penicillin and $100 \mu \mathrm{M}$ streptomycin). Cells were maintained in a humidified cell incubator with $5 \% \mathrm{CO}_{2}$ at $37^{\circ} \mathrm{C}$.

Reagents. Terbinafine (MW, 291.44) was purchased from Sigma-Aldrich (Carlsbad, CA, USA) and dissolved in dimethyl sulfoxide (DMSO) with 5\% Tween 80 (Merck, Darmstadt, Germany). KSR1 siRNA (sc-35762) was purchased from Santa Cruz Biotechnology (Santa Cruz, CA, USA). Transfection of KSR1 siRNA in KB cells was performed using Lipofectamine 2000 (Invitrogen, Carlsbad, CA, USA) according to the manufacturer's instructions.

RNA isolation and reverse transcriptase-polymerase chain reaction (RT-PCR). Total RNA was isolated from cells using the RNeasy Mini Kit (Biomed, Beijing, China). First strand cDNA was reverse transcribed with $1 \mu \mathrm{g}$ total RNA, using the TaKaRa Reverse Transcription Kit (TaKaRa Dalian, Dalian, China) and oligo (dT)-15 primers (TaKaRa). The KSR1 primers used were: 5'-AGCAAGTCCCATGAGTCTCA-3' (sense) and 5'-CAACCTGCAATGCTTGCACT-3' (antisense). GAPDH was used as an internal normalization control. The GAPDH primers used were: 5'-GAAGGCTGGGGCTCATTT-3' (sense) and 5'-GGGGCCATCCACAGTCTT-3' (antisense). PCR amplification of cDNA was performed in reaction volumes of $15 \mu \mathrm{l}$. Finally, products were resolved by $1 \%$ agarose gel electrophoresis, and visualized by ethidium bromide staining and a UV imaging system (UVP, Upland, CA, USA).

Colony formation assay. Cells were seeded at 200 cells per well in 24-well tissue culture plates. After $24 \mathrm{~h}$, cells were treated with various concentrations of terbinafine $(0,30,60$, $90,120,150 \mu \mathrm{g} / \mu \mathrm{l})$. DMSO was used as a control. Three weeks later, colonies were stained with $0.05 \%$ crystal violet containing 50\% methanol, and then counted in 4 to 5 random fields under a microscope at a $100 \mathrm{x}$ magnification. The $\mathrm{IC}_{50}$ value for terbinafine was also determined, and was applied to $\mathrm{KB}$ cells for $0,6,12,24,48$, and $72 \mathrm{~h}$. The optimal concentration and treatment time for terbinafine was used in subsequent experiments.

MTT assay. Cell viability was determined using the 3(4,5-dimethylthiazolyl)- 2,5-diphenyltetrazoliumbromide (MTT) assay (Sigma, Carlsbad, CA, USA). Following treatment with terbinafine, siRNA, or terbinafine plus siRNA, $\mathrm{KB}$ cells were plated in 96-well plates ( 1,000 cells per well). After $24 \mathrm{~h}$, cells were treated with $0.5 \mathrm{mg} / \mathrm{ml}$ MTT for $4 \mathrm{~h}$, and lysed with DMSO. Absorbance rates were measured at 550-560 nm using a microplate reader (Bio-Rad, Hercules, CA, USA).

Detection of apoptosis. Cells were trypsinized, washed twice with cold PBS, and resuspended in $200 \mu$ l binding buffer. Annexin V-FITC was added to a final concentration of $0.5 \mu \mathrm{g} / \mathrm{ml}$ (KeyGEN, Nanjing, China) and incubated at room temperature in the dark. After $20 \mathrm{~min}, 400 \mu \mathrm{l}$ binding buffer was added, and samples were immediately analyzed on a FACSCalibur flow cytometer (Becton Dickinson Medical Devices, Shanghai, China).

Preparation of proteins and ligand structures for docking. We applied our approach to three target proteins: KSR, Raf, and the KSR-MEK1 heterodimer. The structures with crystallographic resolutions of less than $3.0 \AA$ were retrieved from the Protein Data Bank (http://www.rcsb.org). The PDB codes of the selected proteins are: 1KBF---KSR [19], 1FAQ--Raf [20], and 2Y4I---KSR-MEK1 [21]. The molecular structure of terbinafine (CID_1549008) was downloaded from Pubchem Compound (http://www.ncbi.nlm.nih.gov/pccompound). Data were imported into the modeling software SYBYL-X 1.3 (Tripos International, St. Louis, MO, USA). All non-protein components such as water molecules, metal ions, and lipids were deleted, and hydrogen atoms were added to the protein structures. The interaction of terbinafine and proteins was analyzed by SYBYL-X 1.3.

Antibodies and Western blot analysis. Cells were lysed in RIPA buffer (150 mM NaCl, $2 \mathrm{mM}$ EDTA, 1\% Nonidet P-40, $50 \mathrm{mM} \mathrm{NaF}, 1 \mathrm{mM} \mathrm{Na}_{3} \mathrm{VO}_{4}, 1 \mathrm{mM} \mathrm{Na}_{2} \mathrm{MoO}_{4}, 10 \mu \mathrm{M}$ aprotinin, $10 \mu \mathrm{M}$ leupeptin) on ice. Proteins were separated on $10 \%$ SDS-polyacrylamide gel electrophoresis and transferred to nitrocellulose membranes. Membranes were blocked in 5\% milk-TBST and incubated with anti-Raf, anti-phospho-Raf, anti-ERK, anti-phospho-ERK, anti-MEK, and anti-phosphoMEK primary antibodies (Santa Cruz). Peroxidase-coupled anti-mouse IgG, anti-rabbit IgG, and anti-goat IgG secondary antibodies were from Amersham Biosciences (Needham, MA, USA). Bands were visualized by enhanced chemiluminescence (Amersham Pharmacia, Piscataway, NJ, USA).

Subjects. Surgical specimens from 57 patients with OSCC were obtained from the Department of Oral and Maxillofacial Surgery, School of Stomatology, China Medical University from January 2006 to November 2011. None of the patients underwent radiotherapy or chemotherapy before operation. This study was in compliance with the Helsinki Declaration, and was approved by our University Ethics Committee. All patients approved the use of tumor tissues for clinical research. Adjacent mucosa and primary tumors of all the cases were fixed in $4 \%$ formaldehyde solution, embedded in paraffin, and cut into $4 \mu \mathrm{m}$ sections.

Immunohistochemical staining (IHC). IHC of $4-\mu \mathrm{m}$ sections of paraffin-embedded specimens was performed using the rabbit anti-KSR1 polyclonal antibody (Santa Cruz). Briefly, after deparaffinization and hydration, the endogenous peroxidase activity was quenched by a 30 -min incubation in a mixture of $0.3 \%$ hydrogen peroxide solution in $100 \%$ methanol. The sections were blocked for $2 \mathrm{~h}$ at room temperature with $1.5 \%$ blocking serum (Santa Cruz) in PBS and incubated with anti-KSR1 antibody (1:200 dilution) at $4{ }^{\circ} \mathrm{C}$ in a moist chamber overnight, followed by incubation with Envision reagent (DAKO, Carpinteria, CA, USA) and color development in 3,3'-diaminobenzidine tetrahydrochloride (DAB, Sigma). The slides were then lightly counterstained with hematoxylin, dehydrated with ethanol, cleaned with 
A Terbinafine-KSR

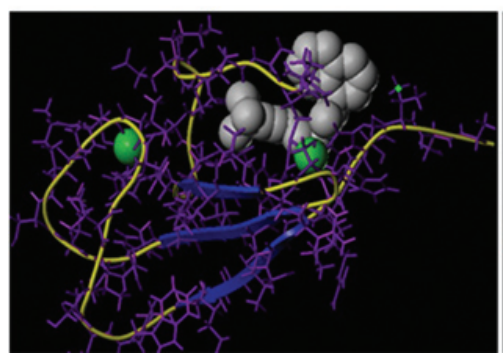

B Terbinafine-KSR-MEK1

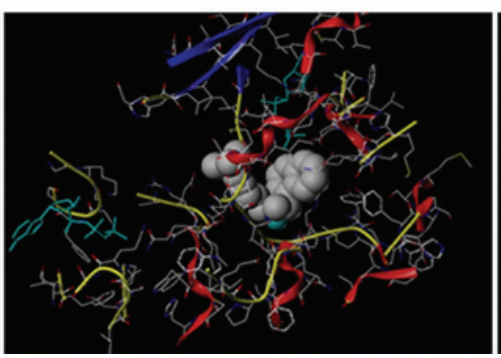

C Terbinafine-Raf

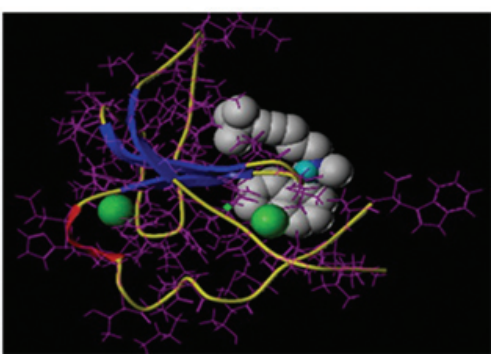

Figure 1. Possible terbinafine-interacting proteins simulated by the SYBYL-X 1.3 modeling software. (A) Position of interaction sites in a model of terbinafine bound to the KSR1 domain. (B) Interaction of terbinafine with the KSR2-MEK1 heterodimer. (C) Interaction of terbinafine with Raf.
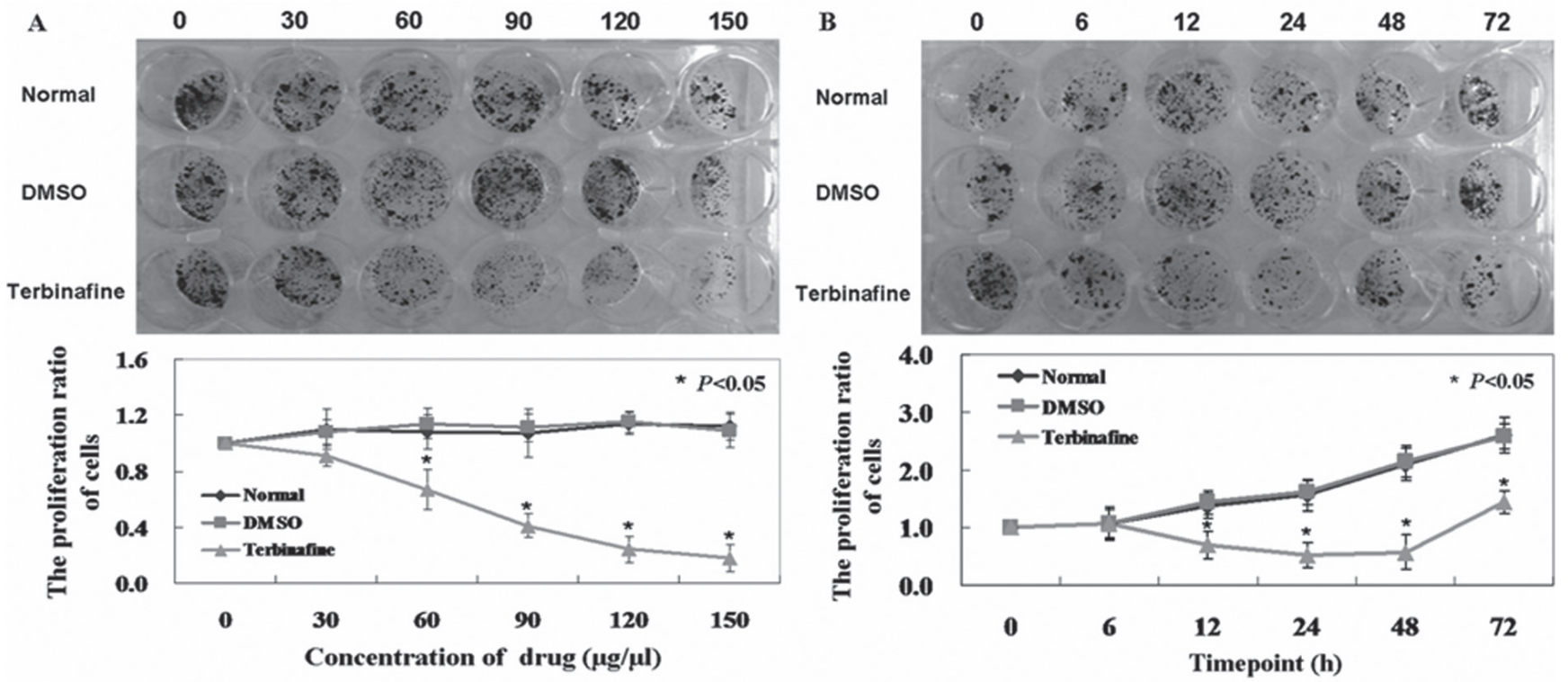

Figure 2. Terbinafine inhibits the growth of KB cells. (A) Growth curves of KB cells treated with different concentrations of terbinafine. (B) Growth curves of $\mathrm{KB}$ cells treated with the $\mathrm{IC}_{50}$ concentration of terbinafine $(85 \pm 3.12 \mu \mathrm{g} / \mu \mathrm{l})$ at different time points. Normal: KB cells; DMSO: KB cells treated with DMSO; Terbinafine: KB cells treated with terbinafine.

xylene, and mounted. Adjacent noncancerous tissues (taken from the farthest margin of oral cancerous tissues) were used as controls. Sections treated without primary antibodies were used as negative controls. The positive percentage of counted cells was graded semi-quantitatively according to a four-tier scoring system: negative (-), 0 5\%; weakly positive $(+)$, $6 \sim 25 \%$; moderately positive $(++), 26 \sim 50 \%$; and strongly positive (+++), 51 100\%.

Statistical analysis. Data were analyzed using GraphPad Prism 5. Significant differences between KSR1 and clinicopathological parameters were computed by chi-square statistics. Kaplan-Meier survival plots were generated and comparisons between survival curves were made with the log-rank statistic. Data from other experiments were expressed as the mean \pm SD (standard deviation) from a representative experiment. P-values less than 0.05 were considered statistically significant.

\section{Results}

Terbinafine is predicted to bind KSR1. To explore the possible proteins that could interact with terbinafine, we applied the modeling software SYBYL-X 1.3, and found that terbinafine docked onto KSR1. Figure 1A shows the predicted binding sites between terbinafine and KSR1. Further analysis demonstrated that terbinafine also interacted with the KSR1MEK1 heterodimer (Fig. 1B). Interestingly, terbinafine could also dock onto Raf, a downstream target of KSR1 signaling (Fig. 1C). Other proteins in the Raf-MEK-ERK signaling pathway showed no interaction with terbinafine (data not 

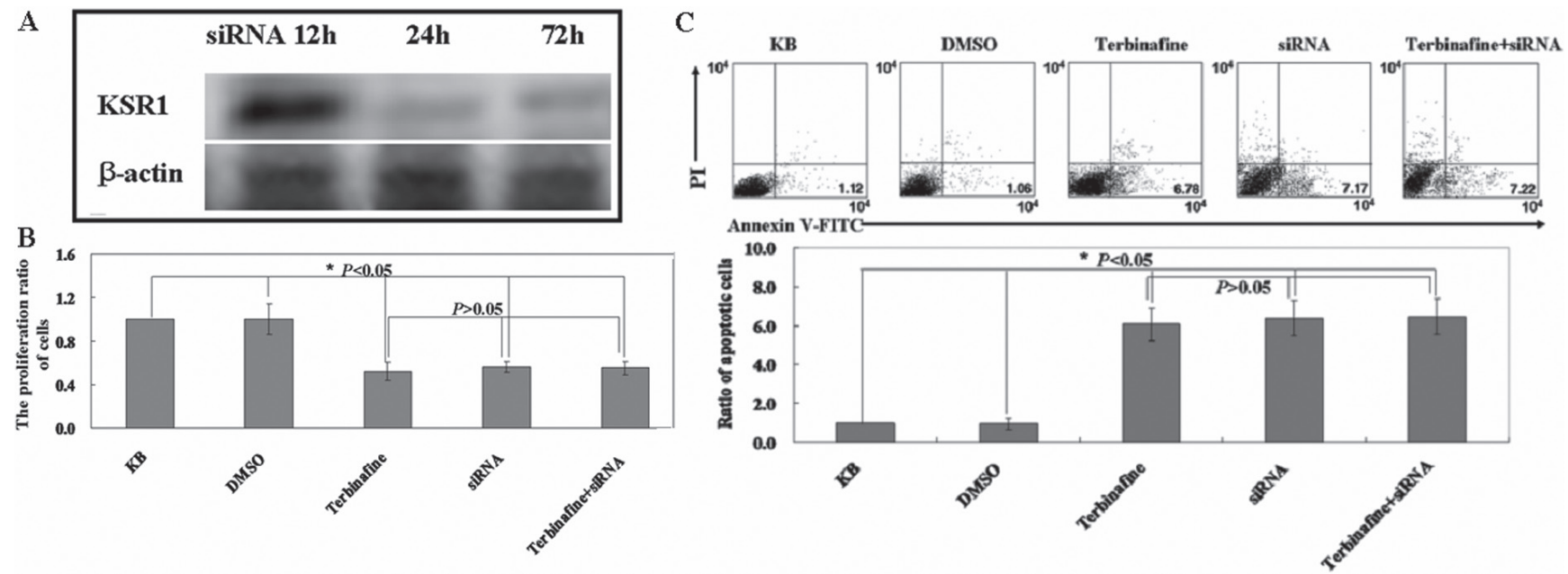

Figure 3. Downregulation of KSR1 leads to reduced cell proliferation and enhanced cell apoptosis. (A) KRS1 protein levels were determined by Western blotting at the indicated time points. $\beta$-actin was used as a loading control. (B) The proliferation ratio of cells was determined by the MTT assay. (C) Apoptotic cells double stained with Annexin V-FITC and PI were detected by flow cytometry. Normal: KB cells; DMSO: KB cells treated with DMSO; Terbinafine: KB cells treated with terbinafine; siRNA: KB cells transfected with KRS1 siRNA; siRNA+ Terbinafine: KB cells both treated with terbinafine and siRNA.

shown). This suggests that terbinafine might inhibit the RafMEK-ERK signaling pathway via direct interaction with KSR1 and/or Raf.

Terbinafine and KSR1 siRNA inhibit the growth of KB cells. To support our above observation, we evaluated cell growth following terbinafine treatment in $\mathrm{KB}$ cells in parallel with siRNA-mediated knockdown of KSR1. The colony formation assay showed that terbinafine significantly decreased the proliferation of $\mathrm{KB}$ cells in a concentration and time-dependent manner (Fig. $2, \mathrm{P}<0.05$ ). In addition, the $\mathrm{IC}_{50}$ value for terbinafine at $48 \mathrm{~h}$ was $85 \pm 3.12 \mu \mathrm{g} / \mu \mathrm{l}$ (Fig. $2 \mathrm{~B}$ ). KSR1 was efficiently downregulated by siRNA (Fig. 3A), which resulted in similar effects on KB cell proliferation after terbinafine treatment, as determined by the MTT assay (Fig. 3B). We additionally treated $\mathrm{KB}$ cells with terbinafine $24 \mathrm{~h}$ after transfection of KSR1 siRNA, and found that there was no significant difference in proliferation of $\mathrm{KB}$ cells treated with siRNA, terbinafine, or siRNA plus terbinafine (Fig. 3B, P > 0.05). We further examined the apoptosis of KB cells treated with siRNA, terbinafine, or siRNA plus terbinafine by FITC-Annexin $V$ and PI double staining. The percentage of apoptotic KB cells after treatment with terbinafine was $6.78 \%$, which was significantly higher than that of untreated cells $(1.12 \%)$ (Fig. 3C, $\mathrm{P}<0.05)$. Similar to proliferation, there was no apparent difference in apoptosis following treatment with siRNA, terbinafine, or siRNA plus terbinafine. These results indicate that terbinafine inhibits cell proliferation and induces apoptosis, mostly likely by facilitating Raf binding to KSR1.

Terbinafine decreases the protein levels of KSR1 and MAKP signaling. KSR1 is an upstream regulator of MAPK signaling. To determine if inhibition of KSR1 leads to suppression of MAPK signaling, we examined the phospho-

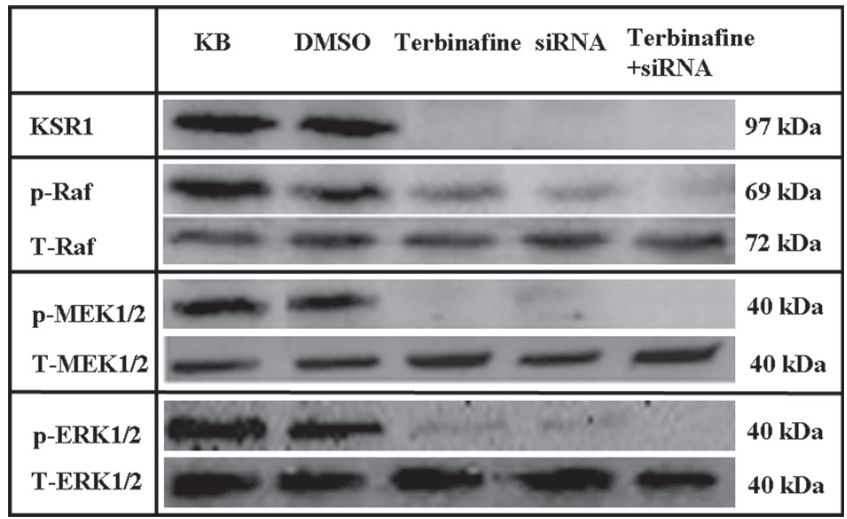

Figure 4. Terbinafine inhibits the Raf/MEK/ERK signaling pathway. Levels of total Raf, MEK 1/2, and ERK 1/2, and their corresponding phosphorylation forms in cell lysates were determined by Western blot analysis.

rylation states of Raf, MEK, and ERK by immunoblotting. KSR1 protein levels decreased in KB cells after treatment with terbinafine (Fig. 4). While total levels of Raf, MEK, and ERK remained unchanged, the levels of phospho-Raf, phospho-MEK, and phospho-ERK were significantly lower in terbinafine-treated cells compared to untreated cells (Fig. 4). Similarly, KSR1 siRNA resulted in decreased phospho-Raf, phospho-MEK, and phospho-ERK signals (Fig. 4). Thus, targeting KSR1 by terbinafine or siRNA downregulates the RAF/MEK/ERK signaling pathway.

Correlation between KSR1 expression and clinicopathological features in OSCC. The MAPK pathway is one of the major cell-growth promoting signaling pathways, and 


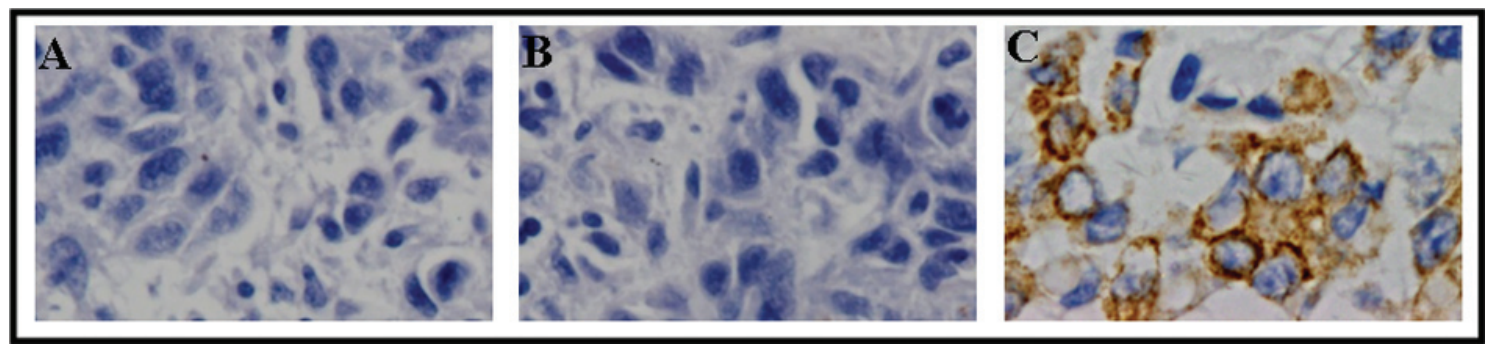

Figure 5. KSR1 is overexpressed in oral squamous cell carcinoma. Representative two paired OSCC and corresponding noncancerous tissues by immunohistochemical staining with anti-KSR1 antibody. (A) Negative control. (B) Paired noncancerous tissue. (C) Cancer tissue. The nuclei were counterstained with hematoxylin.

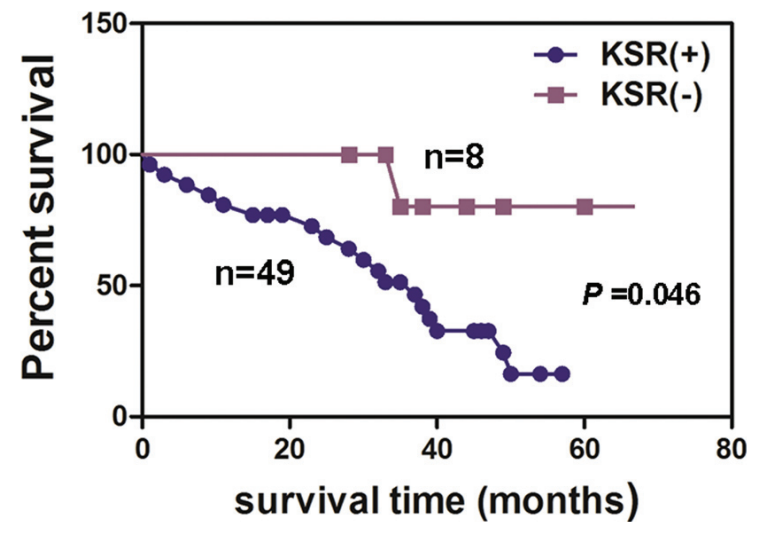

Figure 6. Kaplan-Meyer curve analysis of the association between KSR1 expression and survival time of OSCC patients. The pink line indicates patients with tumors without KSR1 expression, while the purple line represents patients with tumors overexpressing KSR1. The difference was significant in a log-rank test $(P=0.046)$.

most cancer cells depend on this pathway for survival. Our above in vitro data demonstrate that OSCC KB cells rely on KSR1 for proliferation and survival, suggesting that KSR1 signaling may play an important role in the tumorigenesis and maintenance of OSCC. To test this hypothesis, we compared the protein levels of KSR1 with the clinicopathology of OSCC. KSR1 protein was highly expressed in the tumor parts of specimens (Fig. 5). The relationship between KSR1 and the clinicopathological characteristics of these patients are summarized in Table 1. No correlation was found with sex, age, drinking habits, differentiation, lymphatic invasion, venous invasion, and tumor location $(\mathrm{P}>0.05)$. However, KSR1 expression was significantly associated with the clinical staging of OSCC and the smoking habits of patients ( $\mathrm{P}$ $<0.05$ ).

Univariate survival analysis. The survival time of patients in this study ranged from 1 to 60 months, with a median time of 31.8 months. Kaplan Meyer survival analysis demonstrated a clear difference in patients with and without KSR1 expression (Fig. 6) $(\mathrm{P}=0.046)$. The average overall survival of patients with KSR1 overexpressing tumors was
21 months, compared to 54 months in patients with tumors of low KSR1 expression.

\section{Discussion}

In this study, we performed a novel computer simulation analysis (small molecular docking technology), and found that KSR1 is a potential target of terbinafine. The computer simulation results were consistent with our in vitro results. Terbinafine inhibited cell proliferation and induced apoptosis of human OSCC KB cells. In accordance with our findings, a recent study showed that terbinafine inhibits OSCC growth through decreasing cancer cell proliferation [18]. Furthermore, we found that terbinafine reduced KSR1 protein levels in KB cells. Since KSR1 interacts with several proteins that possess kinase activity, such as Raf, MEK1, MAPK, and C-TAK1 [22-26], disruption of KSR1 expression or kinase activity may block activation of ERK1/2, NF-kB, and Akt/PKB [27, 28]. Consistent with previous studies, we found that phospho-Raf, phospho-MEK, and phospho-ERK levels decreased upon KSR1 down-regulation, which was further supported by siRNA-mediated knockdown of KSR1. KSR1 siRNA inhibited the growth of $\mathrm{KB}$ cells in vitro. Interestingly, terbinafine showed no additive effects on KB cells in which KSR1 was decreased by siRNA. Collectively, these results suggest that terbinafine targets KSR1 and induces apoptosis in KB cells via suppressing the Raf/MEK/ERK signaling pathway.

KSR1 is highly conserved from Drosophila and Caenorhabditis elegans to humans [8]. We found KSR1 levels were elevated in OSCC tissues compared to adjacent noncancerous tissues, and KSR1 expression was significantly associated with clinical staging. Our study indicated that KSR1 might be an important factor in the pathogenesis of OSCC. A previous study demonstrated that smoking is a major risk factor for OSCC [4]. In this study, we found that KSR1 expression was higher in smokers than in non-smokers. However, whether smoking is the cause of KSR1 up-regulation remains unclear.

In summary, in vitro, terbinafine down-regulated KSR1 and inhibited the growth of OSCC cells by suppressing the Raf-MEK-ERK signaling pathway. In vivo, KSR1 expression was significantly associated with clinical staging of OSCC and 
Table 1. Relationship between KSR1 expression and clinicopathological parameters of OSCC

\begin{tabular}{|c|c|c|c|c|c|c|c|c|}
\hline \multirow{2}{*}{ Clinicopathological features } & \multirow{2}{*}{$\mathrm{n}$} & \multicolumn{7}{|c|}{ KSR1 expression } \\
\hline & & - & + & ++ & +++ & PR (\%) & $\chi^{2}$ value & $P$ \\
\hline Sex & & & & & & & 1.82 & .324 \\
\hline Female & 28 & 3 & 15 & 5 & 5 & $89.3 \%$ & & \\
\hline Male & 29 & 5 & 13 & 8 & 3 & $82.8 \%$ & & \\
\hline Age(years) & & & & & & & 5.59 & .075 \\
\hline$<60$ & 33 & 4 & 13 & 11 & 5 & $87.9 \%$ & & \\
\hline$\geq 60$ & 24 & 4 & 15 & 2 & 3 & $83.3 \%$ & & \\
\hline Smocking habit & & & & & & & 25.11 & .007 \\
\hline No & 12 & 7 & 2 & 2 & 1 & $41.7 \%$ & & \\
\hline Yes & 45 & 1 & 26 & 11 & 7 & $97.8 \%$ & & \\
\hline Drinking habit & & & & & & & 3.45 & .186 \\
\hline No & 26 & 3 & 11 & 6 & 6 & $88.5 \%$ & & \\
\hline Yes & 31 & 5 & 17 & 7 & 2 & $83.9 \%$ & & \\
\hline Location & & & & & & & 4.64 & .285 \\
\hline Tongue & 22 & 1 & 10 & 7 & 4 & $95.5 \%$ & & \\
\hline Floor of mouth & 13 & 2 & 6 & 3 & 2 & $84.6 \%$ & & \\
\hline Buccal mucosa & 9 & 2 & 5 & 1 & 1 & $77.8 \%$ & & \\
\hline Gingiva & 13 & 3 & 7 & 3 & 1 & $84.6 \%$ & & \\
\hline Clinical Staging & & & & & & & 6.43 & .024 \\
\hline Stage I & 15 & 3 & 6 & 4 & 2 & $80.0 \%$ & & \\
\hline Stage II & 13 & 1 & 5 & 4 & 3 & $92.3 \%$ & & \\
\hline Stage III & 12 & 1 & 9 & 1 & 1 & $91.7 \%$ & & \\
\hline Stage IV & 17 & 3 & 7 & 5 & 2 & $82.4 \%$ & & \\
\hline Differentiation & & & & & & & 1.18 & .349 \\
\hline Well or Moderate & 27 & 5 & 13 & 5 & 4 & $81.5 \%$ & & \\
\hline Poor & 30 & 3 & 15 & 8 & 4 & $90.0 \%$ & & \\
\hline Lymphatic invasion & & & & & & & 2.39 & .092 \\
\hline- & 23 & 2 & 14 & 4 & 3 & $91.3 \%$ & & \\
\hline+ & 34 & 6 & 14 & 9 & 5 & $82.4 \%$ & & \\
\hline Venous invasion & & & & & & & 5.37 & .067 \\
\hline- & 32 & 5 & 19 & 6 & 2 & $84.4 \%$ & & \\
\hline+ & 25 & 3 & 9 & 7 & 6 & $88.0 \%$ & & \\
\hline
\end{tabular}

Abbreviations: PR positive rate, $\chi^{2}$ value chi-squared distribution.

the smoking habits of patients. The cumulative survival rate of patients without KSR1 expression was significantly higher than those with KSR1 overexpression, suggesting the potential development of terbinafine for the treatment of OSCC.

Acknowledgments: We thank Zhou Ning for his insightful comments and technical support.

\section{References}

[1] JEMAL A, BRAY F, CENTER MM, FERLAY J, WARD E et al. Global cancer statistics. CA Cancer J Clin 2011; 61: 69-90. http://dx.doi.org/10.3322/caac.20107

[2] RAO DN, GANESH B, RAO RS, DESAI PB. Risk assessment of tobacco, alcohol and diet in oral cancer-a case control study. Int J Cancer 1994; 58: 469-473. http://dx.doi.org/10.1002/ ijc. 2910580402
[3] IARC. Betel-quid and areca nut chewing and some related nirtosamines. IARC Monogr Eval Carcinog Risks Hum 2003; 85: 11-18.

[4] BALARAJAN R, YUEN P. British smoking and drinking habits: variations by birth. Community Med 1986; 8: 237-239.

[5] ZUR HAUSEN H. Papillomavirus infections-a major cause of human cancers. Biochim Biophys Acta 1996; 1288: F55-78.

[6] FIELD JK. Oncogenes and tumor-suppressor genes in squamous cell carcinoma of the head and neck. Eur J Cancer B Oral Oncol 1992; 28B: 67-76.

[7] MURUGAN AK, MUNIRAJAN AK, TSUCHIDA N. Ras oncogenes in oral cancer: the past 20 years. Oral Oncol 2012; 48: 383-392. http://dx.doi.org/10.1016/j.oraloncology.2011.1 $\underline{2.006}$

[8] CLAPERON A, THERRIEN M. KSR and CNK: two scaffolds regulating RAS-mediated RAF activation. Oncogene 2007; 26: 3143-3158. http://dx.doi.org/10.1038/sj.onc.1210408 
[9] CHANG L, KARIN M. Mammalian MAP kinase signaling cascades. Nature 2001; 410: 37-40. http://dx.doi.org/10.1038/ $\underline{35065000}$

[10] KORTUM RL, LEWIS RE. The molecular scaffold KSR1 regulates the proliferative and oncogenic potential of cells. Mol Cell Biol 2004; 24: 4407-4416. http://dx.doi.org/10.1128/ MCB.24.10.4407-4416.2004

[11] LlOBET D, ERITJA N, DOMINGO M, BERGADA L, MIRANTES C, et al. KSR1 is overexpressed in endometrial carcinoma and regulates proliferation and TRAIL-induced apoptosis by modulating FLIP levels. Am J Pathol. 2011; 178: 1529-1543. http://dx.doi.org/10.1016/ j.ajpath.2010.12.041

[12] ALY R, FORNEY R, BAYLES C. Treatments for common superficial fungal infections. Dermatol Nurs 2001; 13: 91-101.

[13] DE DONCKER P, GUPTA AK. Itraconazole and terbinafine in perspective: from petri dish to patient. Postgrad Med; 1999; 6-11.

[14] FAERGEMANN J, ZEHENDER H, DENOUEL J, MILLERIOUX L. Levels of terbinafine in plasma, stratum corneum, dermis-epidermis (without stratum corneum), sebum, hair and nails during and after $250 \mathrm{mg}$ terbinafine orally once per day for four weeks. Acta Derm Venereol 1993; 73: 305-309.

[15] HOSSEINI-YEGANEH M, MCLACHLAN AJ. Tissue distribution of terbinafine in rats. J Pharm Sci 2001; 90: 1817-1828. http://dx.doi.org/10.1002/jps.1132

[16] LEE WS, CHEN RJ, WANG YJ, TSENG H, JENG JH et al. In vitro and in vivo studies of the anticancer action of terbinafine in human cancer cell lines: G0/G1 p53-associated cell cycle arrest. Int J Cancer 2003; 106: 125-137. http://dx.doi. org/10.1002/ijc.11194

[17] YANG KC, WU CC, WU CH, CHEN JH, CHU CH et al. Involvement of proapoptotic Bcl-2 family members in terbinafine-induced mitochondrial dysfunction and apoptosis in HL60 cells. Food Chem Toxicol 2006; 44: 214-226. http:// dx.doi.org/10.1016/j.fct.2005.07.008

[18] CHIEN MH, LEE TS, KAO C, YANG SF, LEE WS. Terbinafine inhibits oral squamous cell carcinoma growth through anticancer cell proliferation and anti-angiogenesis. Mol Carcinog 2012; 51: 389-399. http://dx.doi.org/10.1002/mc.20800
[19] ZHOU M, HORITA DA, WAUGH DS, BYRD RA, MORRISON DK. Solution structure and functional analysis of the cysteine-rich C1 domain of kinase suppressor of Ras (KSR). J Mol Biol 2002; 315: 435-446. http://dx.doi.org/10.1006/ imbi.2001.5263

[20] MOTT HR, CARPENTER JW, ZHONG S, GHOSH S, BELL $\mathrm{RM}$ et al. The solution structure of the Raf-1 cysteine-rich domain: a novel ras and phospholipid binding site. Proc Natl Acad Sci U S A 1996; 93: 8312-8317. http://dx.doi. org/10.1073/pnas.93.16.8312

[21] BRENNAN DF, DAR AC, HERTZ NT, CHAO WC, BURLINGAME AL et al. A Raf-induced allosteric transition of KSR stimulates phosphorylation of MEK. Nature 2011; 472: 366-369. http://dx.doi.org/10.1038/nature09860

[22] THERRIEN M, MICHAUD NR, RUBIN GM, MORRISON DK. KSR modulates signal propagation within the MAPK cascade. Genes Dev 1996; 10: 2684-2695. http://dx.doi. org/10.1101/gad.10.21.2684

[23] YU W, FANTL WJ, HARROWE G, WILLIAMS LT. Regulation of the MAP kinase pathway by mammalian Ksr through direct interaction with MEK and ERK. Curr Biol 1998; 8: 56-64. http://dx.doi.org/10.1016/S0960-9822(98)70020-X

[24] DENOUEL-GALY A, DOUVILLE EM, WARNE PH, PAPIN C, LAUGIER D et al. Murine Ksr interacts with MEK and inhibits Ras-induced transformation. Curr Biol 1998; 8: 46-55. http://dx.doi.org/10.1016/S0960-9822(98)70019-3

[25] XING H, KORNFELD K, MUSLIN AJ. The protein kinase KSR interacts with 14-3-3 protein and Raf. Curr Biol 1997; 7: 294-300. http://dx.doi.org/10.1016/S0960-9822(06)00152-7

[26] MULLER J, ORY S, COPELAND T, PIWNICA-WORMS $\mathrm{H}$, MORRISON DK. C-TAK1 regulates Ras signaling by phosphorylating the MAPK scaffold, KSR1. Mol Cell 2001; 8: 983-993. http://dx.doi.org/10.1016/S1097-2765(01)00383-5

[27] YAN F, JOHN SK, POLK DB. Kinase suppressor of Ras determines survival of intestinal cells exposed to tumor necrosis factor. Cancer Res 2001; 61: 8668-8675.

[28] YAN F, POLK DB. Probiotic bacterium prevents cytokineinduced apoptosis in intestinal epithelial cells. J Biol Chem 2002; 277: 50959-50965. http://dx.doi.org/10.1074/jbc. $\underline{\mathrm{M} 207050200}$ 